\title{
Fatal Gastrointestinal Bleeding Probably Caused by an Aortoduodenal Fistula Following Surgical Repair of an Inflammatory Abdominal Aortic Aneurysm during Postoperative Steroid Therapy
}

\author{
Akihiko Ikeda*, Toru Tsukada, Taisuke Konishi, Kanji Matsuzaki and Tomoaki Jikuya
}

Department of Cardiovascular Surgery, Tsukuba Medical Center Hospital, Tsukuba, Japan

\begin{abstract}
We describe a case of fatal gastrointestinal bleeding probably caused by an aortoduodenal fistula following open surgical repair of an inflammatory abdominal aortic aneurysm (AAA) during postoperative steroid therapy. A 71-year old man underwent a graft replacement for an inflammatory AAA. Prolonged elevated C-reactive protein and newly emerged ascites after surgery were improved by oral administration of predonine. The steroid therapy had been continued until 5 months after surgery, when he suffered from fatal gastrointestinal bleeding. We speculated that the gastrointestinal bleeding was caused by an aortoduodenal fistula and discuss the etiology of this condition.
\end{abstract}

Keywords: Inflammatory abdominal aortic aneurysm; Aortoduodenal fistula; Steroid

\section{Introduction}

Inflammatory abdominal aortic aneurysm (AAA) is characterized by markedly thickened aneurysmal walls and adhesion to adjacent organs. It is generally thought that the inflammatory process of the disease can be resolved by surgical treatment. Rarely, however, cases with progression of inflammation or newly developed complications in preoperatively uninvolved organs have been reported [1,2]. We herein report a case of open surgical repair of an inflammatory AAA, which complicated with prolonged elevated $\mathrm{C}$-reactive protein (CRP) and newly emerged ascites after surgery. Although both disorders were improved by steroid therapy, our patient suffered from fatal gastrointestinal bleeding presumably due to an aortoduodenal fistula.

\section{Case Report}

A 71-year old man, who complained of abdominal pain, was referred to our hospital. Computed tomography $(\mathrm{CT})$ revealed an infrarenal AAA with a maximal diameter of $80 \mathrm{~mm}$ at the level of the right common iliac artery. The density of fat around the aneurysm was high, which suggested the presence of inflammation (Figure 1A). Laboratory examination showed that the inflammatory response was elevated, with a white blood cell (WBC) count of $11000 / \mu \mathrm{l}$ and CRP of $19.3 \mathrm{mg} / \mathrm{dl}$, though he had no fever. We performed emergent surgery because of a diagnosis of impending rupture of the aneurysm.
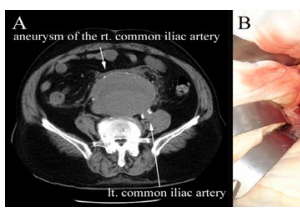

Figure 1: Preop histopathology.

A. Preoperative abdominal CT. The maximal diameter of the aneurysm is $80 \mathrm{~mm}$ at the level of the right common iliac artery. The density of the postperitoneal fat around the aneurysm is elevated.

B. Gross appearance. The post-peritoneum, which is red and thickened, widely adheres to the aneurysm.

C. Histological examination of the resected aneurysmal wall. Inflammatory infiltration of lymphocytes observed in the adventitia.
In the supine position, a full-laparotomy was made. The postperitoneum, which was red and thickened, widely adhered to the aneurysm (Figure 1B), though fortunately, the duodenum did not. A graft interposition using a Dacron graft (Gelsoft Plus $16 \mathrm{~mm} \times 8 \mathrm{~mm}$, Vaskutek Ltd, Inchinnan, Renfrewshire, Scotland) with right internal iliac aneurysmorrhaphy was performed without any problems. Histological examination of the resected aneurysmal wall revealed that inflammatory infiltration of the lymphocytes existed in thickened adventitia with fibrosis, which was compatible with an inflammatory aneurysm (Figure 1C). Bacterial examination did not detect any microorganisms.

After surgery, WBC was normalized, but CRP was not improved and remained at approximately $15 \mathrm{mg} / \mathrm{dl}$. Because the patient had no fever, we did not test blood culture. CT showed that the remnant aneurysmal wall was enhanced and high peri-aortic fat density remained, though there was no obvious sign of graft infection. Furthermore, 30day postoperatively, the patient complained of loss of appetite and abdominal distension due to ascites (Figures $2 \mathrm{~A}$ and $2 \mathrm{~B}$ ). The causes of transudative ascites, such as hypoalbuminemia, liver dysfunction, and portal hypertension, were not detected. Although an abdominal CT was performed again, there was no remarkable change, except the newly emerged ascites. Thereby, we considered that inflammation of the remnant aneurysmal wall and peri-aortic region were still persistent, and decided to start steroid therapy, which consisted of the oral administration of predonine at $20 \mathrm{mg} /$ day. The steroid therapy drastically improved the patient's condition and the dose of predonine was gradually decreased to $5 \mathrm{mg} /$ day, however, it was returned to 10

*Corresponding author: Akihiko Ikeda, Department of Cardiovascular Surgery Tsukuba Medical Center Hospital, 1-3-1 Amakubo, Tsukuba, Ibaraki 305-8558, Japan, Tel: +81-29-851-3511; Fax: +81-29-851-3511; E-mail: ai-cvs@sd6.so-net.ne.jp

Received December 26, 2013; Accepted January 09, 2014; Published January 11,2014

Citation: Ikeda A, Tsukada T, Konishi T, Matsuzaki K, Jikuya T (2014) Fatal Gastrointestinal Bleeding Probably Caused by an Aortoduodenal Fistula Following Surgical Repair of an Inflammatory Abdominal Aortic Aneurysm during Postoperative Steroid Therapy. J Vasc Med Surg 2: 123 doi: 10.4172/2329 6925.1000123

Copyright: ( 2014 Ikeda A, et al. This is an open-access article distributed unde the terms of the Creative Commons Attribution License, which permits unrestricted use, distribution, and reproduction in any medium, provided the original author and source are credited. 
Citation: Ikeda A, Tsukada T, Konishi T, Matsuzaki K, Jikuya T (2014) Fatal Gastrointestinal Bleeding Probably Caused by an Aortoduodenal Fistula Following Surgical Repair of an Inflammatory Abdominal Aortic Aneurysm during Postoperative Steroid Therapy. J Vasc Med Surg 2: 123 doi: 10.4172/2329-6925.1000123

$\mathrm{mg} /$ day because of re-elevation of CRP (Figure 3). Finally, he was discharged with oral administration of predonine at $10 \mathrm{mg} /$ day on the 43rd postoperative day. Four-month postoperatively, CRP decreased to $0.99 \mathrm{mg} / \mathrm{dl}$ with continuous administration of predonine at $10 \mathrm{mg} /$ day, though the enhancement of the remnant aneurysmal wall and high peri-aortic fat densities were still observed on CT (Figures 2C and 2D).

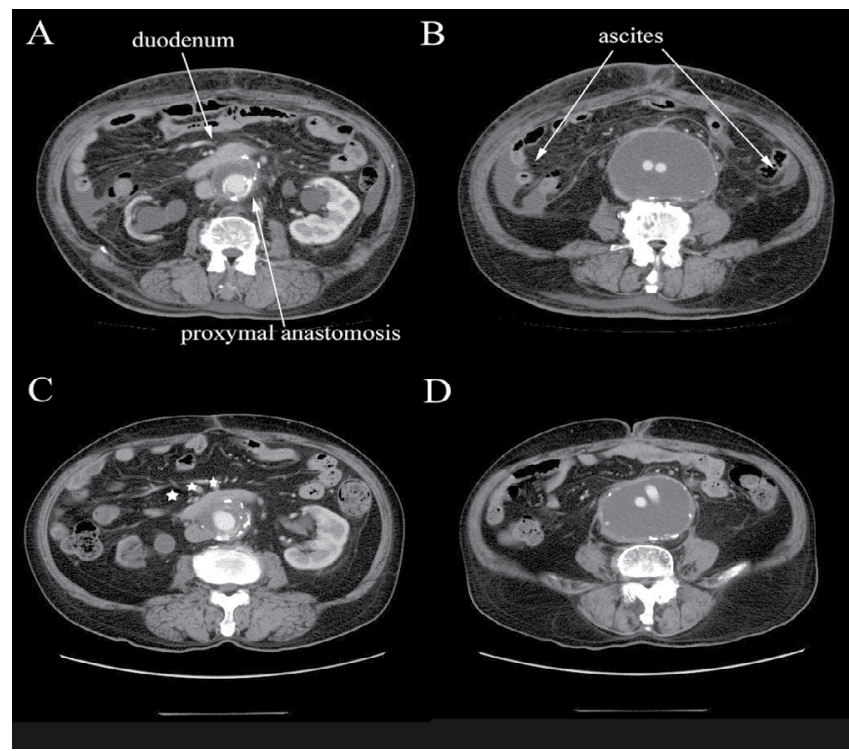

Figure 2: Abdominal CT before and after steroid therapy.

$A$ and $B$ : Abdominal CT before steroid therapy. The remnant aneurysmal wall around the graft is enhanced and peri-aortic fat density is high. In addition, ascites is observed.

C and D: Abdominal CT after steroid therapy. (CRP indicated $0.99 \mathrm{mg} / \mathrm{dl}$.) Ascites disappeared, but inflammatory signs of the remnant aneurysmal wall remained. The duodenum adhered to the remnant aneurysmal wall (Stars indicates this portion).

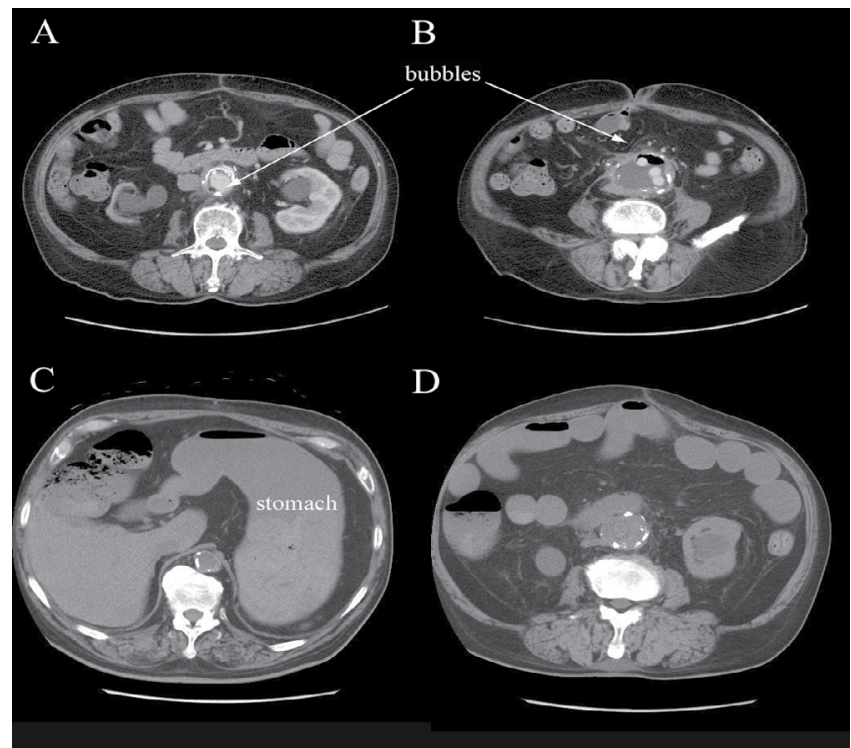

Figure 3: Relation between inflammatory response and dose of predonine. Prolonged elevated CRP maintained after surgery, however, it was dramatically improved by steroid therapy. When dose of predonine was decreased to $5 \mathrm{mg} /$ day, the value of CRP elevated to $14.4 \mathrm{mg} / \mathrm{dl}$.

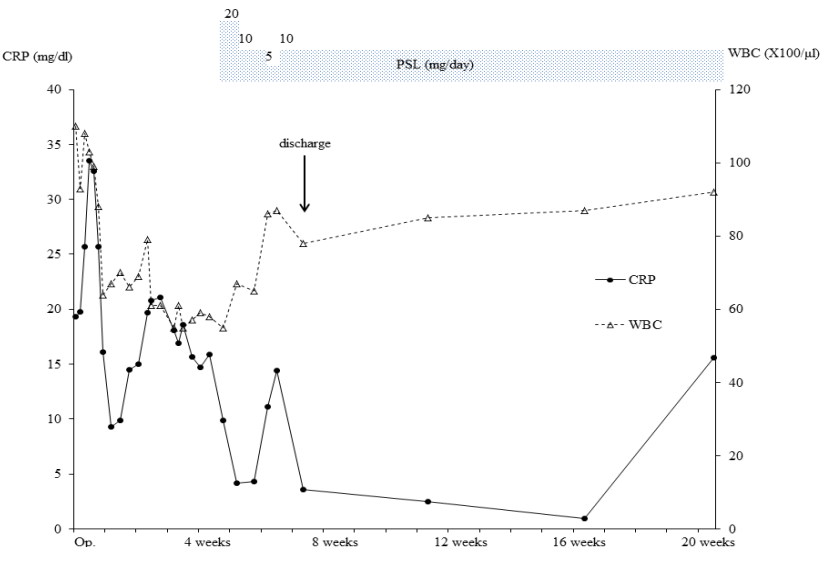

Figure 4: Pre and post-mortal abdominal CT.

$A$ and B: Abdominal CT just before cardiac arrest. Peri-graft space is dramatically shrunken with bubble formation.

C and D: post-mortal abdominal CT. Alimentary tract is filled with a massive amount of blood.

Five-month postoperatively, however, the patient came to our emergency room complaining of fever, abdominal pain and diarrhea. Laboratory examination, revealed that inflammatory response was re-elevated (WBC: $9200 / \mu \mathrm{l}, \mathrm{CRP}: 15.6 \mathrm{mg} / \mathrm{dl}$ ). CT showed that the peri-graft space was dramatically reduced and was accompanied by bubble formation (Figure 4A and 4B). Although we immediately began antibiotic therapy and scheduled further examinations, the patient suddenly vomited a massive amount of blood and went into cardiac arrest. We performed cardiopulmonary resuscitation, but could not save his life. Post-mortal CT showed that the gastrointestinal tract was filled with a massive amount of blood and that there was no bleeding into the retroperitoneal space (Figures $4 \mathrm{C}$ and $4 \mathrm{D}$ ). On the basis of preand post-mortal CT findings, we speculated that a fistula had formed between the duodenum and peri-graft space, which subsequently caused the graft infection leading to final fatal bleeding from the anastomosis into the duodenum.

\section{Discussion}

Inflammatory AAA, which was first reported by Walker et al., is characterized by marked thickening of the aneurysmal wall and strong adhesion to adjacent organs. Histologically, marked thickening of the adventitia with fibrosis and infiltration of inflammatory cells, mainly lymphocytes, are observed [3]. The inflammatory process can affect and obstruct the duodenum, ureters, left renal vein, inferior vena cava, and rarely, the small bowel or colonic mesentery [4]. The etiology of inflammatory AAA remains uncertain, though several theories, such as an autoimmune disease, a chronic viral infection, and a variation of the inflammatory process of atherosclerosis, have been previously discussed [5].

The treatment of inflammatory AAA involves surgical repair, endovascular stent grafting and steroid therapy. Surgical repair of inflammatory AAA, which is commonly a prosthetic graft replacement, is conventional. Although there are technical problems which are associated with adherence to adjacent structures, the results compare favorably with common atherosclerotic AAA [6]. In most cases, a prosthetic graft replacement not only prevents rupture, but also regresses the inflammatory process. However, there are a few cases with persistent inflammation after surgery or postoperative development 
Citation: Ikeda A, Tsukada T, Konishi T, Matsuzaki K, Jikuya T (2014) Fatal Gastrointestinal Bleeding Probably Caused by an Aortoduodenal Fistula Following Surgical Repair of an Inflammatory Abdominal Aortic Aneurysm during Postoperative Steroid Therapy. J Vasc Med Surg 2: 123 doi: $10.4172 / 2329-6925.1000123$

Page 3 of 3

of organ complications in preoperatively uninvolved structures, and it has been reported that postoperative steroid therapy improved complications caused by a persistent or progressive inflammatory process after surgery [1,2]. In our case, CT showed that the enhancement of the remnant aneurysmal wall and high peri-aortic fat density remained after surgery, which apparently suggested persistent inflammation of the remnant aneurysmal wall. On the contrary, no other abdominal organs showed inflammatory changes. Furthermore, our patient did not have other causes of transudative ascites. Thereby, we considered that the elevated CRP and ascites were caused by persistent inflammation of the remnant aneurysmal wall, and performed steroid therapy. Actually, ascites disappeared and the value of CRP fluctuated depending of dose of predonine (Figure 3 ). These facts suggested that postoperative steroid therapy was effective in our case.

Nevertheless, our patient died of gastrointestinal bleeding. Although an autopsy could not be performed, we speculated that a fistula between the duodenum and peri-graft space was formed, subsequently, the peri-graft space dramatically shrunk and the graft infection occurred. Finally, our patient suffered from fatal bleeding from the anastomosis to the duodenum.

Aortoenteric fistula, which often causes massive gastrointestinal bleeding, is divided into two groups, such as primary and secondary. Primary aortoenteric fistula is a communication between the native aorta and gastrointestinal tract. While secondary aortoenteric fistula arises from the suture line after abdominal aortic reconstructive surgery, especially using a prosthetic graft [7]. The etiology of secondary aortoenteric fistula after prosthetic aortic grafting includes direct contact with the prosthetic graft or the site of anastomosis, pseudoaneurysm formation caused by failure of anastomosis, and graft infection.

In our case, there were two possible causes of a fistula between the duodenum and peri-graft space. One was the local persistent inflammation of the remnant aneurysmal wall. On the basis of a series of CT findings, the prosthetic graft did not contact with the duodenum directly. Before the graft infection, the duodenum adhered to the enhanced aneurysmal wall located around the graft (Figures 2C). Such cases have never been reported, although cases of an aortoduodenal fistula following endovascular repair of inflammatory AAA have been reported [8]. Furthermore, primary aortoenteric fistula without aortic aneurysms is caused by inflammation of the aorta itself, such as septic aortitis [7].

The other potential cause was a duodenal ulcer which was one of the side effects of steroid, however, the risk of upper gastrointestinal complications induced by steroid is still debated [9]. In addition, we administered "low" dose of predonine. Our patient did not take NSAIDs, but took PPI and also the duodenal ulcer prefers to affect the bulbus. The cases with peptic ulcer induced aortoenteric fistula have been previously reported [10]. Most cases affected the esophagus and stomach, rarely duodenum, and had the gastrointestinal disorders except peptic ulcer, such as the hiatus hernia and after surgery of the gastrointestinal tract. The reports about an aortoduodenal fistula caused by only peptic ulcer are rare and outdated.

In conclusion, we experienced a case of inflammatory AAA complicated with fatal gastrointestinal bleeding probably caused by an aortoduodenal fistula. The pathological process of inflammatory AAA is rarely persistent after surgery, in such cases, steroid therapy is effective. However, it might not be able to completely resolve persistent local inflammation, attention should be given to any newly emerged complications, especially, in cases which CT shows the presence of inflammation of the remnant aneurysmal wall.

\section{Acknowledgements}

The authors are grateful to Mr. Avi Landau for his language consultation.

\section{References}

1. Stotter AT, Grigg MJ, Mansfield AO (1990) The response of perianeurysmal fibrosis-the "inflammatory" aneurysm-to surgery and steroid therapy. Eur J Vasc Surg 4: 201-205.

2. von Fritschen U, Malzfeld E, Clasen A, Kortmann H (1999) Inflammatory abdominal aortic aneurysm: a postoperative course of retroperitoneal fibrosis. J Vasc Surg 30: 1090-1098.

3. Walker DI, Bloor K, Williams G, Gillie I (1972) Inflammatory aneurysms of the abdominal aorta. Br J Surg 59: 609-614

4. Goldstone J, Malone JM, Moore WS (1978) Inflammatory aneurysms of the abdominal aorta. Surgery 83: 425-430.

5. Kasashima S, Zen Y, Kawashima A, Endo M, Matsumoto Y, et al. (2009) A new clinicopathological entity of IgG4-related inflammatory abdominal aortic aneurysm. J Vasc Surg 49: 1264-1271.

6. Sterpetti AV, Hunter WJ, Feldhaus RJ, Chasan P, McNamara M, et al. (1989) Inflammatory aneurysms of the abdominal aorta: Incidence, pathologic, and etiologic considerations. J Vasc Surg 9: 643-649.

7. Ranasinghe W, Loa J, Allaf N, Sebastian MG (2011) Primary aortoenteric fistulae: the challenges in diagnosis and review of treatment. Ann Vasc Surg 25: 386.e1-386.e5.

8. Parry DJ, Waterworth A, Kessel D, Robertson I, Berridge DC, et al. (2001) Endovascular repair of an inflammatory abdominal aortic aneurysm complicated by aortodudenal fistulation with an unusual presentation. J Vasc Surg 33: 874879

9. Fardet L, Kassar A, Cabane J, Flahault A (2007) Corticosteroid-induced adverse events in adults: frequency, screening and prevention. Drug Saf 30: 861-881.

10. Odze RD, Begin LR (1991) Peptic-ulcer-induced aortoenteric fistula. report of a case and review of the literature. J Gastroenterol 13: 682-686. 